\title{
Fibrinogen: A Feasible Biomarker in Identifying the Severity and Acute Exacerbation of Chronic Obstructive Pulmonary Disease
}

Mikash Mohan ${ }^{1}$, Ashwaghosha Parthasarathi ${ }^{2}$, Chaya S K ${ }^{1}$, Jayaraj Biligere Siddaiah ${ }^{1}$, Padukudru A. Mahesh ${ }^{1}$

1. Department of Pulmonology, Jagadguru Sri Shivarathreeshwara Medical College, Mysore, IND 2. Department of Epidemiology and Public Health, Allergy Asthma and Chest Center, Mysore, IND

Corresponding author: Padukudru A. Mahesh, mahesh1971in@yahoo.com

\section{Abstract}

\section{Background}

Chronic obstructive pulmonary disease (COPD) is no longer considered a disease exclusive to the respiratory system. It is a multipronged disease with both lung and systemic involvement. Although the forced expiratory volume (FEV) in one second is one of the most commonly used markers to assess disease severity, in recent years, biomarkers such as interleukin-1 beta, serum C-X-C motif chemokine ligand 10, fibrinogen, soluble receptor for advanced glycation, surfactant protein $\mathrm{D}$, and club cell secretory protein have been proven to be effective markers to assess disease severity.

\section{Objective}

The current study aimed to test the association of fibrinogen levels with increased exacerbation of COPD per year and lower lung function and to discuss its potential utility as a biomarker.

\section{Methodology}

A total of 105 participants were enrolled in the study. The study participants included 35 stable COPD patients, 35 COPD patients with acute exacerbation, and 35 non-COPD healthy controls (matched for age and gender). All patients above 18 years of age who were diagnosed with COPD as per the Global Initiative for Chronic Obstructive Disease (GOLD) guidelines were considered for inclusion in the study. The patients were divided into stable COPD group and acute exacerbations of COPD (AECOPD) group based on the Anthonisen criteria. Sociodemographic factors, six-minute walk test, Medical Research Council Dyspnea Scale, and COPD Assessment Test scale were computed. Spirometry according to the American Thoracic Society guidelines and hematological investigations including serum fibrinogen were performed. Additionally, GOLD staging and severity indices were used to determine the clinical phenotyping of COPD, namely, ADO (age, dyspnea, airflow obstruction) index, BODE (body mass index, airflow obstruction, dyspnea, and exercise capacity) index, and DOSE (dyspnea, obstruction, smoking, exacerbation) index.

Review began 06/27/2021 Review ended 07/28/2021 Published 08/03/2021

๑) Copyright 2021 Mohan et al. This is an open access article distributed under the terms of the Creative Commons Attribution License CC-BY 4.0., which permits unrestricted use, distribution, and reproduction in any medium, provided the original author and source are credited.

\section{Results}

Plasma fibrinogen level was significantly higher in the COPD groups compared to the control group. Plasma fibrinogen level was elevated in AECOPD compared to stable COPD patients. In addition, fibrinogen levels showed a positive correlation with important functional indices and prognostic markers such as BODE, ADO, and DOSE indices and a negative correlation with lung function. The odds of predicting an acute exacerbation of COPD for patients with FEV of $<50 \%$ and FEV of $>50 \%$ were 17.2 (area under the curve [AUC] $=0.825$; sensitivity $=90.4 \%$; specificity $=62.79 \%)$ and $15.1(\mathrm{AUC}=0.791$; sensitivity $=57.7 \%$; specificity $=$ $92.5 \%$, respectively.

\section{Conclusions}

Plasma fibrinogen has the potential to be an important biomarker in the management of COPD and its exacerbation due to its ability to be responsive to the COPD disease statuses such as the severity of COPD and AECOPD.

\section{Categories: Pulmonology}

Keywords: fibrinogen, inflammation, copd, aecopd, biomarker

\section{Introduction}

Chronic respiratory diseases such as chronic obstructive pulmonary disease (COPD) are among the leading causes of morbidity and mortality worldwide [1,2]. Globally, it is estimated that $5 \%$ of all deaths are attributed to COPD, of which more than $90 \%$ occur in low- and middle-income countries (LMICs) [2]. COPD 
is no longer considered a disease exclusive to the respiratory system. It is a multipronged disease with both lung and systemic involvement [3].

Forced expiratory volume in one second (FEV1) is the marker most commonly associated with COPD. However, FEV1 is known to correlate inadequately with symptoms of COPD. This is mainly because the FEV1 values are based on the severity of airflow obstruction which is known to inaccurately predict the occurrence of exacerbations [4].

COPD, especially in the latter stages of the disease, is frequently associated with exacerbations. Acute exacerbation of COPD (AECOPD) is defined as "an acute worsening of respiratory symptoms requiring a change in treatment" [5]. These exacerbations are a known contributor to diminished lung function, quality of life, and mortality rates [6-8], and the prevention of COPD exacerbations is a vital therapeutic goal. Recent systematic reviews on the association of protein biomarkers with COPD outcomes identified the emergence of interleukin-1 beta, serum C-X-C motif chemokine ligand 10, fibrinogen, soluble receptor for advanced glycation, surfactant protein D, and club cell secretory protein as novel biomarkers [9]. Plasma fibrinogen levels are of great importance, especially in LMICs, as they are economically feasible, easy to measure, and repeated as needed in routine clinical practice [10]. The goal of this study was to evaluate the association of serum fibrinogen levels with COPD outcomes and the effectiveness of serum fibrinogen levels and their optimal cut-off values as prognostic biomarkers for hospitalized COPD patients.

The current study aimed to test the hypothesis that elevated fibrinogen levels are associated with increased AECOPD cases per year, lower lung function, and lower functional indices such as BODE (body mass index, airflow obstruction, dyspnea, and exercise capacity), ADO (age, dyspnea, airflow obstruction), and DOSE (dyspnea, obstruction, smoking, exacerbation). In addition, it tests the effectiveness of serum fibrinogen levels and their optimal cut-off values to predict episodes of acute exacerbation for hospitalized COPD patients with moderate and severe COPD.

\section{Materials And Methods \\ Study population}

Participants were recruited from a tertiary care university teaching hospital from November 2019 to April 2020. The Institutional Ethics Committee of JSS Medical College, Mysore approved this study. This study included 35 stable COPD patients, 35 COPD patients with acute exacerbation, and 35 non-COPD healthy controls, who were matched for both age and gender.

All patients above 18 years of age who were diagnosed with COPD as per the Global Initiative for Chronic Obstructive Disease (GOLD) guidelines were considered for inclusion in the study. The patients were divided into stable COPD group and AECOPD group based on the Anthonisen criteria, which comprise three patientreported items, namely, increased dyspnea, increased sputum volume, and increased sputum purulence [11].

Patients were excluded from the study if they failed to give consent; had any other respiratory disease other than COPD; were diagnosed with disseminated intravascular coagulopathy, pulmonary embolism, or deep vein thrombosis; or were on regular oral steroids and anticoagulants. Additionally, for the stable COPD group, patients who were currently stable but had an acute exacerbation within the previous month were excluded.

\section{Laboratory and clinical assessments}

Sociodemographic factors such as age, gender, body mass index (BMI), history of asthma exacerbation, and history of smoking were gathered from patient files. The six-minute walk test was performed on the day of the outpatient department (OPD) visit for those with stable COPD, while the AECOPD patients were tested on the day of discharge. Medical Research Council Dyspnea Scale (mMRC) and COPD Assessment Test (CAT) scale were used to assess the respiratory symptoms on the day of admission or OPD visit recorded by the resident physician $[12,13]$.

On the day of admission or the OPD visit, $2 \mathrm{~mL}$ of venous blood sample was drawn and data from a hematological panel, conducted using an automated blood analyzer Sysmex XN 1000 (Sysmex Corp., Kobe, Japan), were recorded. In addition, absolute neutrophile count/absolute lymphocyte count and PLR platelet count/absolute lymphocyte count were calculated. Serum fibrinogen levels were assessed by turbidimetric immunoassay using KinesisDx kit (KinesisDx, Brea, CA) (assay range: 0.6-9.6 mg/mL) according to the standard protocols. Spirometry was performed on the same day using EasyOne ${ }^{\circledR}$ spirometer (NDD Medical Technologies, Zurich, Switzerland) according to American Thoracic Society guidelines [14].

Comorbidities in the patient group were recorded using the Charlson comorbidity index (CCI) and ageadjusted CCI [15]. Additionally, GOLD staging and severity indices were used to determine the clinical phenotyping of COPD, namely, ADO index, BODE index, and DOSE index [16]. 


\section{Statistical analysis}

Statistical analysis was done using Jamovi (v1.6, The jamovi project). Continuous variables were presented as either mean \pm standard deviation (SD). Categorical variables were presented as percentages. Statistical significance between categorical variables was assessed using the chi-square test and by Student's t-test for continuous variables. Correlation between plasma fibrinogen levels and functional indices such as BODE, $\mathrm{ADO}$, and DOSE was calculated using Pearson's correlation coefficient. Patients were further stratified into those with fibrinogen levels of $<350 \mathrm{mg} / \mathrm{dL}$ and $>350 \mathrm{mg} / \mathrm{dL}$. This threshold was based on an integrated database at the participant level from five individual studies conducted by the COPD Biomarkers Qualification Consortium [17].

The area under the curve (AUC), sensitivity, specificity, odds ratio (OR), and optimal cut-off values were calculated based on the receiver operating characteristic (ROC) curve for groups with moderate (FEV1 of $>50 \%$ ) and severe (FEV1 of $<50 \%$ ) COPD. A p-value of $<0.05$ was considered statistically significant.

\section{Results}

A total of 105 patients were enrolled in the study. The patients were divided into three groups: healthy controls, stable COPD patients, and AECOPD patients, with 35 participants in each group. The mean age of controls was $59.5 \pm 13.1$, while the mean ages of stable COPD and AECOPD patients were $60.7 \pm 9.87$ and 63.7 \pm 9.28 years. The study sample consisted predominantly of males $(n=101 ; 95.2 \%)$. Overall, $85.8 \%(n=91)$ participants had a smoking history. The age-adjusted CCI score was $7.23 \pm 1.75$ and $3.37 \pm 3.75$ in the stable COPD group and $3.33 \pm 1.63$ in the control group.

Lung function was more impaired in the AECOPD than the stable COPD group, as assessed using percentage predicted FEV1 (stable COPD $=62.3 \pm 17.1$; AECOPD $=50.6 \pm 15.8 ; \mathrm{p}=0.004$ ). Total leukocyte count, neutrophils, and fibrinogen levels were higher in the AECOPD group $(\mathrm{p}<0.001)$. Further details on the study population are listed in Table 1. Plasma fibrinogen level in the control group was the lowest at $267 \pm 37.2$ $\mathrm{mg} / \mathrm{dL}$ followed by $353 \pm 32.2 \mathrm{mg} / \mathrm{dL}$ in stable COPD and $405 \pm 71.6 \mathrm{mg} / \mathrm{dL}$ in AECOPD groups (Figure 1a). Similarly, fibrinogen levels were the lowest in GOLD stage 1 patients $(286 \pm 51.7 \mathrm{mg} / \mathrm{dL})$ and the highest in stage $4(403 \pm 108 \mathrm{mg} / \mathrm{dL})$ (Figure $1 b)$. 


\section{Cureus}

\begin{tabular}{|c|c|c|c|}
\hline \multirow{2}{*}{ COPD group } & Controls $(n=35)$ & Stable COPD ( $=35)$ & AECOPD (n = 35) \\
\hline & Mean \pm SD & Mean \pm SD & Mean \pm SD \\
\hline Age & $59.5 \pm 13.1$ & $60.7 \pm 9.94$ & $63.8 \pm 9.4$ \\
\hline Gender (M/F) & $33 / 2$ & $34 / 1$ & $32 / 3$ \\
\hline BMI & $25.5 \pm 3.87$ & $21.5 \pm 4.25$ & $22.4 \pm 3.44$ \\
\hline \multicolumn{4}{|l|}{ Smoking history } \\
\hline Nonsmoker & 35 (100\%) & 3 (8.57\%) & $1(2.85 \%)$ \\
\hline Current smoker & 0 & $32(91.42 \%)$ & $34(97.14 \%)$ \\
\hline \multicolumn{4}{|l|}{ Charlson comorbidity index } \\
\hline Unadjusted & $2.01 \pm 1.04$ & $2.03 \pm 2.06$ & $3.97 \pm 1.07$ \\
\hline Age-adjusted & $3.33 \pm 1.63$ & $3.37 \pm 3.75$ & $7.23 \pm 1.75$ \\
\hline \multicolumn{4}{|l|}{ Pulmonary function tests } \\
\hline 6MWD (m) & N/A & $439 \pm 36.5$ & $330 \pm 88.3$ \\
\hline FEV1 (L) & $2.28 \pm 0.58$ & $1.34 \pm 0.465$ & $1.15 \pm 0.37$ \\
\hline FEV1 (\%) & $91 \pm 13.3$ & $60.7 \pm 17$ & $51.4 \pm 14.6$ \\
\hline FEV1 (\% predicted) & $90.3 \pm 14.1$ & $62.3 \pm 17.1$ & $50.6 \pm 15.8$ \\
\hline FEV1/FVC (\%) & $97.3 \pm 4.94$ & $73.7 \pm 9.57$ & $68.0 \pm 11.8$ \\
\hline FEV1/FVC (\% predicted) & $98.1 \pm 7.91$ & $76 \pm 9.34$ & $67.9 \pm 10.3$ \\
\hline \multicolumn{4}{|l|}{ Hematological tests } \\
\hline Hemoglobin (g/dL) & $13.7 \pm 2.33$ & $14.3 \pm 2.31$ & $14.5 \pm 1.93$ \\
\hline Packed cell volume (\%) & $39.8 \pm 5.58$ & $42.8 \pm 8.49$ & $45.5 \pm 6.7$ \\
\hline TLC (cells/mm³) & $7,998 \pm 2,509$ & $9,306 \pm 2,380$ & $12,155 \pm 5,544$ \\
\hline Neutrophils (cells $/ \mathrm{mm}^{3}$ ) & $5,053 \pm 2,219$ & $7,209 \pm 2,873$ & $10,287 \pm 6,089$ \\
\hline Lymphocytes (cells $/ \mathrm{mm}^{3}$ ) & $2,237 \pm 735$ & $1,413 \pm 856$ & $1,334 \pm 784$ \\
\hline Platelets $\left(10^{6} / \mathrm{mm}^{3}\right)$ & $2.7 \pm 0.817$ & $2.47 \pm 0.67$ & $2.51 \pm 0.855$ \\
\hline Fibrinogen (mg/dL) & $267 \pm 37.2$ & $353 \pm 32.2$ & $405 \pm 71.6$ \\
\hline Eosinophils (cells/mm³) & NA & $201 \pm 209$ & $256 \pm 492$ \\
\hline
\end{tabular}

\section{TABLE 1: Baseline characteristics of the study participants.}

COPD: chronic obstructive pulmonary disease; AECOPD: acute exacerbation of chronic obstructive pulmonary disease; BMI: body mass index; FVC: forced vital capacity; FEV1: forced expiratory volume in one second; 6MWD: six-minute walk distance; TLC: total leucocyte count 


\section{Cureus}

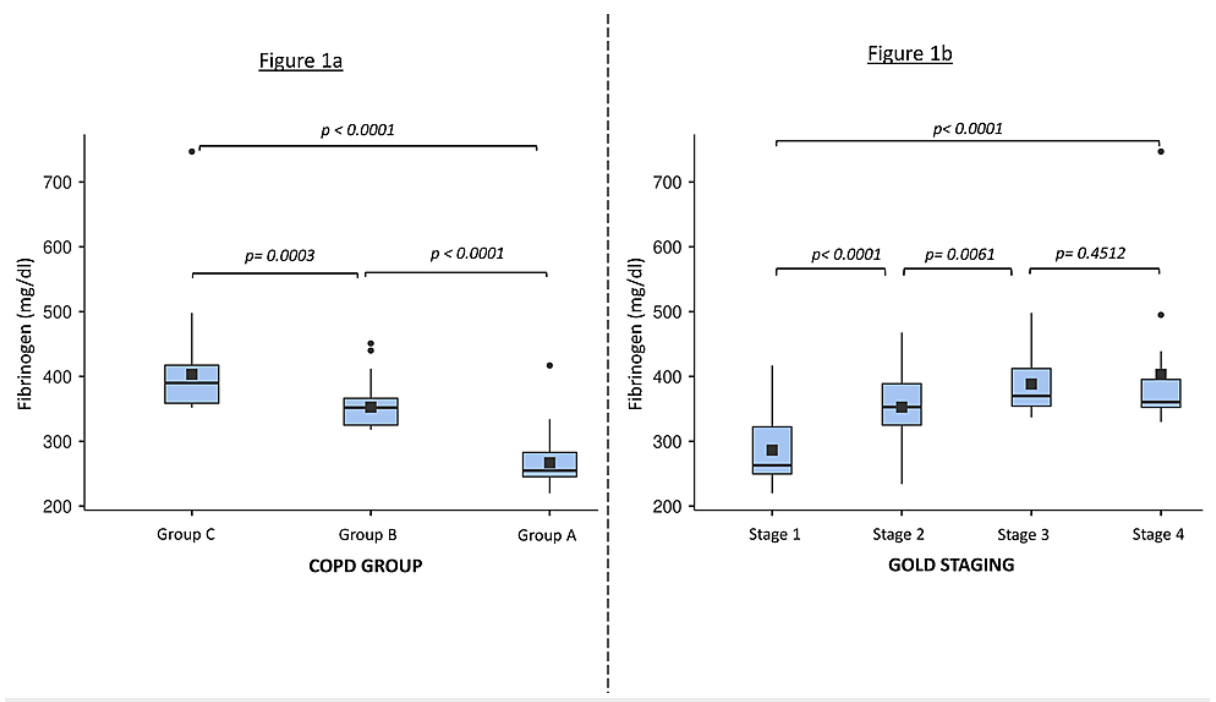

\section{FIGURE 1: Plasma fibrinogen levels in the patient population.}

a: Box plot illustrating plasma fibrinogen levels between different COPD groups. The lower and upper borders of the box plot illustrate the Q1 and Q3 quartile. The solid square in the box illustrates the mean, for which the test of significance was calculated. Data outside the Q1-Q3 range are plotted as dots (outliers of the data). Group A: healthy individuals; Group B: individuals with stable COPD; Group C: individuals with acute exacerbation of COPD.

b: Box plot illustrating plasma fibrinogen levels between different COPD groups differentiated by GOLD staging. The lower and upper borders of the box plot illustrate the Q1 and Q3 quartiles. The solid square in the box illustrates the mean, for which the test of significance was calculated. Data outside the Q1-Q3 range are plotted as dots (outliers of the data). Stage 1: FEV1, $\geq 80 \%$; Stage 2: FEV1, 50-79\%; Stage 3: FEV1, 3049\%; Stage 4: FEV1: $\leq 30 \%$.

COPD: chronic obstructive pulmonary disease; GOLD: Global Initiative for Chronic Obstructive Disease; FEV1: forced expiratory volume in one second

Binomial multivariable analysis of various risk factors between both the control and the COPD group and the stable COPD and AECOPD group showed fibrinogen as an independent risk factor in both scenarios (adjusted odds ratio: 2.9 [2.27, 3.71]; 1.7 [1.37, 2.12]) (Table 2). 


\section{Cureus}

\begin{tabular}{|c|c|c|}
\hline \multirow{2}{*}{ COPD group } & Model 1 & Model 2 \\
\hline & AOR (95\% Cl) & AOR (95\% Cl) \\
\hline Age & $1.04(0.85-1.28)$ & $1.35(0.88-2.7)$ \\
\hline Gender & $1.48(0.98-2.10)$ & $1.43(0.95-2.03)$ \\
\hline BMI & $1.08(0.68-1.71)$ & $1.10(0.70-1.74)$ \\
\hline Smoking (ref: Y) & $4.39(4.00-4.82)^{\star}$ & $5.10(3.81,6.81)^{\star}$ \\
\hline Age-adjusted CCl & $1.02(0.84,1.24)$ & $2.09(0.9,5.21)$ \\
\hline \multicolumn{3}{|l|}{ Pulmonary function tests } \\
\hline 6MWD (m) & NA & $1.2(0.41,3.47)$ \\
\hline FEV1 (L) & $2.32(1.2,4.5)^{\star}$ & $1.94(1.03,3.67)^{*}$ \\
\hline FEV1/FVC & $3.92(3.58-4.30)^{\star}$ & $3.97(3.62-4.36)^{\star}$ \\
\hline \multicolumn{3}{|l|}{ Hematological tests } \\
\hline Hemoglobin (g/dL) & $1.39(0.71,2.71)$ & $1.65(0.86,3.15)$ \\
\hline Packed cell volume (\%) & $1.03(0.56,1.87)$ & $1.12(0.61,2.04)$ \\
\hline TLC (cells $/ \mathrm{mm}^{3}$ ) & $2.19(1.22-3.91)^{\star}$ & $1.44(0.95,2.19)$ \\
\hline Neutrophils (cells $/ \mathrm{mm}^{3}$ ) & $1.4(0.51,3.88)$ & $2.2(0.88,5.5)$ \\
\hline Lymphocytes (cells/mm³) & $1.01(0.79,1.28)$ & $2.18(0.9,5.29)$ \\
\hline Platelets $\left(10^{6} / \mathrm{mm}^{3}\right)$ & $1.58(0.82,3.02)$ & $1.2(0.74,1.97)$ \\
\hline Fibrinogen (mg/dL) & $2.9(2.27,3.71)^{\star}$ & $1.7(1.37,2.12)^{\star}$ \\
\hline Eosinophils (cells $/ \mathrm{mm}^{3}$ ) & NA & $1.32(0.73,2.37)$ \\
\hline
\end{tabular}

\section{TABLE 2: Binomial multivariable analysis for independent risk for different models.}

Model 1: binomial multivariable analysis was done between healthy controls and COPD patients. Model 2: binomial multivariable analysis was done between stable COPD patients and AECOPD patients.

* indicates a statistically significant outcome with a p-value $<0.05$.

AOR: adjusted odds ratio; BMI: body mass index; CCI: Charlson comorbidity index; 6MWD: six-minute walk distance; FVC: forced vital capacity; FEV1: forced expiratory volume in one second; TLC: total leukocyte count

\section{Patient stratification based on fibrinogen levels}

The patients were divided into two groups based on fibrinogen levels: fibrinogen $<350 \mathrm{mg} / \mathrm{dL}$ ( $\mathrm{n}=51$ ) and fibrinogen $\geqslant 350 \mathrm{mg} / \mathrm{dL}(\mathrm{n}=54)$. No significant difference was observed in age-adjusted CCI $(\mathrm{p}=0.939)$ between the two groups. The high fibrinogen group had a higher mMRC dyspnea grade $(p<0.001)$ and CAT score than the low fibrinogen group $(\mathrm{p}=<0.001)$. The high fibrinogen group included patients with a greater number of yearly acute exacerbations $(\mathrm{p}<0.001)$. GOLD stage $\mathrm{D}$ was the most common in both groups $(\mathrm{n}=$ 33.9\%). The two groups showed significant differences in the GOLD COPD indices $(p=0.029)$. The decline in lung function, comprising FEV1 ( $p=0.017), F V C(p=0.007)$, and distance of the six-minute walk test $(p=$ 0.016 ), was considerably worse in the high fibrinogen group than in the low fibrinogen group. Further details are listed in Table 3. 


\section{Cureus}

\begin{tabular}{|c|c|c|c|}
\hline & Fibrinogen $<350 \mathrm{mg} / \mathrm{dL}(\mathrm{n}=51)$ & Fibrinogen $\geq 350 \mathrm{mg} / \mathrm{dL}(\mathrm{n}=54)$ & \\
\hline & Mean \pm SD & Mean \pm SD & P-value \\
\hline Age-adjusted CCI & $3.63 \pm 3.68$ & $3.58 \pm 2.88$ & 0.939 \\
\hline mMRC scoring & $2.13 \pm 0.51$ & $2.98 \pm 0.98$ & $<0.001$ \\
\hline \multicolumn{4}{|l|}{ GOLD staging } \\
\hline Stage $1(\%)$ & $18 \pm 35.90$ & $10 \pm 17.90$ & \multirow{4}{*}{0.029} \\
\hline Stage $2(\%)$ & $17 \pm 33.30$ & $12 \pm 22.40$ & \\
\hline Stage 3 (\%) & $1 \pm 2.60$ & $2 \pm 4.50$ & \\
\hline Stage 4 (\%) & $14 \pm 28.20$ & $20 \pm 37.30$ & \\
\hline CAT score & $16.9 \pm 9.1$ & $23.7 \pm 10.4$ & $<0.001$ \\
\hline Number of AE per year & $1.6 \pm 0.69$ & $1.9 \pm 0.58$ & $<0.001$ \\
\hline BODE index & $3.4 \pm 1.45$ & $4.11 \pm 1.24$ & 0.009 \\
\hline ADO index & $3.2 \pm 1.42$ & $4.95 \pm 1.4$ & $<0.001$ \\
\hline DOSE index & $2.9 \pm 0.87$ & $4.08 \pm 1.45$ & $<0.001$ \\
\hline FVC (L) & $2.14 \pm 0.68$ & $2.11 \pm 0.44$ & 0.007 \\
\hline FEV1 (L) & $1.35 \pm 0.51$ & $1.23 \pm 0.38$ & 0.017 \\
\hline FEV1/FVC & $0.62 \pm 0.08$ & $0.58 \pm 0.09$ & 0.029 \\
\hline 6MWD (m) & $346 \pm 39.6$ & $329 \pm 67.2$ & 0.016 \\
\hline
\end{tabular}

TABLE 3: Pulmonary findings of patients stratified using plasma fibrinogen levels.

GOLD staging: Stage 1: FEV1, $\geq 80 \%$; Stage 2: FEV1, 50-79\%; Stage 3: FEV1, 30-49\%; Stage 4: FEV1, $\leq 30 \%$.

CCI: Charlson comorbidity index; mMRC: modified Medical Research Council Dyspnea; CAT: COPD Assessment Test; AE: acute exacerbations; FVC: forced vital capacity; FEV1: forced expiratory volume in one second; 6MWD: Six-minute walk distance

\section{Correlation among chronic obstructive pulmonary disease severity and} plasma fibrinogen

In addition, we found a significant positive correlation between fibrinogen level and the number of exacerbations per year $(r=0.27, p=0.005)$ and a negative correlation with FEV1 $(r=0.613, p<0.001)$. The fibrinogen level revealed statistically significant positive correlations with BODE index $(r=0.27, p=0.05)$, ADO index $(r=0.33, p<0.001)$, and DOSE index $(r=0.45, p<0.001)$ (Figure 2$)$. 


\section{Cureus}

Figure 2
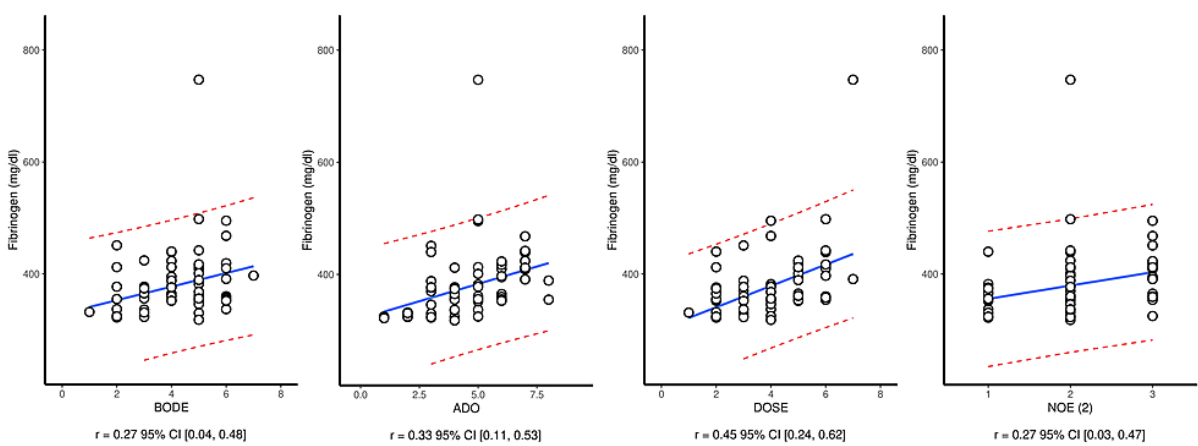

FIGURE 2: Correlation between the COPD severity index and the level of fibrinogen.

COPD: chronic obstructive pulmonary disease; DOSE: dyspnea, obstruction, smoking, exacerbation; BODE: body mass index, airflow obstruction, dyspnea, exercise; ADO: age, dyspnea, airflow obstruction; NOE: number of exacerbations per year

\section{Prognostic accuracy of fibrinogen}

The mean fibrinogen level in stable COPD patients was lower than AECOPD $(353 \pm 31.9 \mathrm{mg} / \mathrm{dL}$ vs $405 \pm 71.1$ $\mathrm{mg} / \mathrm{dL} ; \mathrm{p}<0.01$ ). The overall cut-off for fibrinogen was $358 \mathrm{mg} / \mathrm{dL}$ with a sensitivity of $80 \%$ and specificity of $68.57 \%$ for all COPD patients (AUC $=0.820$; odds of predicting AECOPD $=19.6$ ). Prognostic accuracy was further calculated after being stratified into COPD patients as group A (FEV1 of $<50 \%$ ) with 26 patients and group B (FEV1 of $>50 \%$ ) with 44 patients.

In group A, a fibrinogen cut-off of $353 \mathrm{mg} / \mathrm{dL}$ had a sensitivity of $90.4 \%$ and specificity of $62.79 \%$ (using Youden's index), an AUC of 0.825 , and the odds of 17.2 for predicting acute COPD exacerbations. In group B, the cut-off was higher at $391 \mathrm{mg} / \mathrm{dL}$ with an AUC of 0.791 (sensitivity: $57.7 \%$; specificity: 92.5\%), and the odds of predicting acute COPD exacerbations was 15.1 (Figure 3).

Figure 3

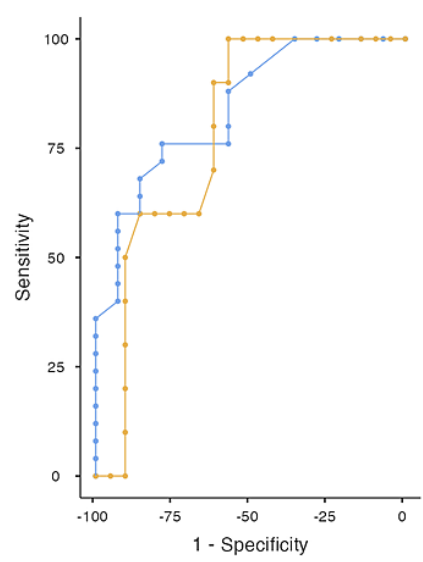

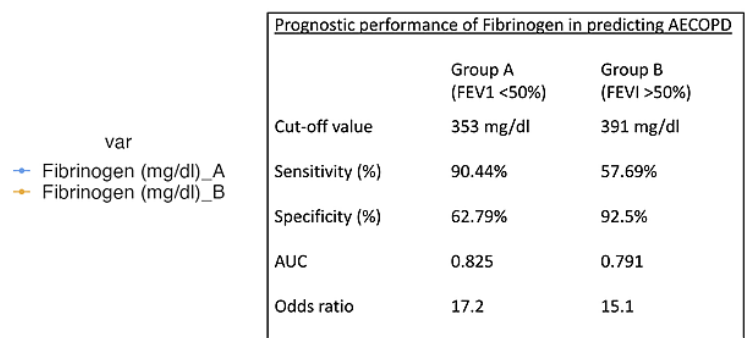

FIGURE 3: ROC analysis for plasma fibrinogen levels for Group A and B. Group A: FEV1 <50\%; Group B: FEV1 > 50\%.

AECOPD: acute exacerbation of chronic obstructive pulmonary disease; FEV1: forced expiratory volume in one second; AUC: area under the curve; ROC: receiver operating characteristic 


\section{Discussion}

We observed that plasma fibrinogen levels were the highest in the AECOPD group and significantly different from those with stable COPD or healthy controls. In addition, plasma fibrinogen levels had a positive correlation with important functional indices and prognostic markers such as the BODE, ADO, and DOSE. The best correlation was with the DOSE index and the least was with the BODE index. Furthermore, elevated fibrinogen levels were associated with an increased number of AECOPD per year and were significantly associated with lower lung function. Significant differences were observed in fibrinogen levels between mild and moderate COPD and moderate and severe COPD, but not between severe and very severe COPD. Fibrinogen levels of $>350 \mathrm{mg} / \mathrm{dL}$ had a $90 \%$ sensitivity but lower specificity to predict AECOPD in severe COPD patients (FEV1 of $<50 \%$ ), while fibrinogen levels of $>390 \mathrm{mg} / \mathrm{dL}$ had more than $90 \%$ specificity to predict AECOPD but had poor sensitivity in moderate COPD patients (FEV1 of >50\%).

Fibrinogen, a major acute-phase reactant, is predominantly synthesized in the liver. Fibrinogen is then converted into fibrin by thrombin for blood coagulation [18]. The synthesis of fibrinogen is upregulated as a response to inflammation which is a key pathophysiology underlying COPD [19]. COPD, a systemic disease, comprises various phenotypes making disease progression and outcome difficult to predict [20-22]. Multiple cohorts including Evaluation of COPD Longitudinally to Identify Predictive Surrogate Endpoints (ECLIPSE), National Health and Nutrition Examination Survey III (NHANES III) study, and Framingham Heart Study Offspring Cohort (FHSOC) concluded that plasma fibrinogen levels can be used as a prognostic biomarker to assess AECOPD [16]. The U.S. Food and Drug Administration and the European Medicines Agency have approved plasma fibrinogen as a prognostic biomarker to assess AECOPD and mortality in COPD confirming its clinical utility [23]. However, it should be noted that the demographics from these large cohorts consisted mainly of the Caucasian population. Because plasma fibrinogen levels are known to vary with demographics and ethnicities, the results from other cohorts may have varied applicability [24]. The mean fibrinogen levels from our study were similar to the NHANES III cohort study but lower than the ECLIPSE study and higher than Atherosclerosis Risk in Communities and Cardiovascular Health Study studies. This can be attributed to the differences in assessment methods for plasma fibrinogen levels or differences in the severity of COPD and exacerbators in the study population or differences in ethnicity [25]. For plasma fibrinogen levels to be accepted as a biomarker, it is crucial to standardize its measurement according to ethnicity and disease severity.

Similar to our study, large-scale longitudinal cohort studies performed in the general population observed that patients with higher plasma fibrinogen levels had increased rates of COPD exacerbations and hospitalization rates during the follow-up period $[26,27]$. We also saw a negative correlation between FEV1 and plasma fibrinogen levels and a positive correlation with various COPD-related indices (BODE, ADO, and DOSE). These findings were congruent with a similar study performed among COPD patients from South Korea [28]. The correlation between plasma fibrinogen and the various prognostic indices were only mild to moderate suggesting that fibrinogen may measure different aspects of the disease. Future studies are needed to confirm whether the addition of fibrinogen to the indices (for example, BODEF) is an even better indicator of disease outcomes.

In addition, plasma fibrinogen levels may be useful in decision-making concerning the consideration of antimicrobial agents in the treatment of AECOPD. Studies observed that a rise in plasma fibrinogen levels was more marked in the presence of symptoms consistent with an infective etiology, especially those of viral etiology [29]. Steroids are an important part of the management of AECOPD, and treatment with steroids has shown a significant decrease in plasma fibrinogen than in patients in nonsteroid therapy [30].

A novel finding of our study is the calculation of predictive accuracy of plasma fibrinogen levels in AECOPD in moderate and severe COPD patients. We found that in both severe (AUC: 0.82; OR: 17.2) and moderate (AUC: 0.79; OR: 15.1) COPD, elevated plasma fibrinogen was found to be significantly associated with risk of exacerbation. Larger and longitudinal studies assessing the use of plasma fibrinogen as a predictive tool for patients with different severities of COPD are required.

Overall, we observed that plasma fibrinogen is independently associated with COPD and its severity, exacerbations, and frequent exacerbators. Though we have not been able to test the responsiveness of the biomarker during stable disease and exacerbations in the same patient, previous studies have demonstrated in longitudinal studies that plasma fibrinogen is responsive to disease stability [22]. This would help place this biomarker in a position to fill an important lacuna.

The GOLD guidelines have a clear strategy on when to escalate the treatment but are not clear on when to de-escalate. Early de-escalation could lead to relapse of symptoms and another exacerbation. There is a need to identify a biomarker that could guide de-escalation. Future longitudinal studies are needed to critically evaluate whether serial plasma fibrinogen levels can be used to inform de-escalation in AECOPD patients.

The main strengths of the study include a well-characterized study population. Diagnosis of COPD and its severity were determined according to the GOLD guidelines. To our knowledge, there is no study conducted in the Indian population investigating the association and predictive power of serum fibrinogen in COPD, its 
severity, and its exacerbation adding to the scientific literature information from a different ethnic population. However, there are a few notable limitations to the study. Our data were from a single center, including relatively small sample size. The distribution of gender in the study was greatly skewed toward males and was reflective of tobacco smoking COPD which may not be representative of the COPD population in India. The subjects were drawn from the OPD and patients admitted to a tertiary care center and may not be reflective of the COPD patients in the community.

\section{Conclusions}

Plasma fibrinogen has the potential to be an important biomarker in the management of COPD and its exacerbations due to its ability to be responsive to COPD disease statuses such as the severity of COPD and AECOPD. Longitudinal studies in real-world clinical situations are needed to assess and understand the relevance of plasma fibrinogen as a biomarker for COPD, disease severity, and exacerbations of COPD, and whether it can guide de-escalation of treatment in AECOPD.

\section{Additional Information \\ Disclosures}

Human subjects: Consent was obtained or waived by all participants in this study. Institutional Ethics Committee of JSS Medical College issued approval JSS/MC/PG/4623/2018-19. Animal subjects: All authors have confirmed that this study did not involve animal subjects or tissue. Conflicts of interest: In compliance with the ICMJE uniform disclosure form, all authors declare the following: Payment/services info: All authors have declared that no financial support was received from any organization for the submitted work. Financial relationships: All authors have declared that they have no financial relationships at present or within the previous three years with any organizations that might have an interest in the submitted work. Other relationships: All authors have declared that there are no other relationships or activities that could appear to have influenced the submitted work.

\section{References}

1. The Lancet: GBD 2017: a fragile world. Lancet. 2018, 392:1683. 10.1016/S0140-6736(18)32858-7

2. WHO. Chronic respiratory diseases. (2021). Accessed: June 14, 2021: https://www.who.int/healthtopics/chronic-respiratory-diseases.

3. Agusti AG: COPD, a multicomponent disease: implications for management . Respir Med. 2005, 99:670-82. 10.1016/j.rmed.2004.11.006

4. Nishimura K, Izumi T, Tsukino M, Oga T: Dyspnea is a better predictor of 5-year survival than airway obstruction in patients with COPD. Chest. 2002, 121:1434-40. 10.1378/chest.121.5.1434

5. Soler-Cataluña JJ, Martínez-García MA, Román Sánchez P, Salcedo E, Navarro M, Ochando R: Severe acute exacerbations and mortality in patients with chronic obstructive pulmonary disease. Thorax. 2005, 60:92531. 10.1136/thx.2005.040527

6. Donaldson GC, Seemungal TA, Bhowmik A, Wedzicha JA: Relationship between exacerbation frequency and lung function decline in chronic obstructive pulmonary disease. Thorax. 2002, 57:847-52. 10.1136/thorax.57.10.847

7. Jinjuvadia C, Jinjuvadia R, Mandapakala C, Durairajan N, Liangpunsakul S, Soubani AO: Trends in outcomes, financial burden, and mortality for acute exacerbation of chronic obstructive pulmonary disease (COPD) in the United States from 2002 to 2010. COPD. 2017, 14:72-9. 10.1080/15412555.2016.1199669

8. Bourbeau J, Ford G, Zackon H, Pinsky N, Lee J, Ruberto G: Impact on patients' health status following early identification of a COPD exacerbation. Eur Respir J. 2007, 30:907-13. 10.1183/09031936.00166606

9. Serban KA, Pratte KA, Bowler RP: Protein biomarkers for COPD outcomes. Chest. 2021, 159:2244-53. 10.1016/j.chest.2021.01.004

10. Bafadhel M, McKenna S, Terry S, et al.: Acute exacerbations of chronic obstructive pulmonary disease: identification of biologic clusters and their biomarkers. Am J Respir Crit Care Med. 2011, 184:662-71. 10.1164/rccm.201104-05970C

11. Anthonisen NR, Manfreda J, Warren CP, Hershfield ES, Harding GK, Nelson NA: Antibiotic therapy in exacerbations of chronic obstructive pulmonary disease. Ann Intern Med. 1987, 106:196-204. 10.7326/00034819-106-2-196

12. Mahler DA, Wells CK: Evaluation of clinical methods for rating dyspnea . Chest. 1988, 93:580-6. 10.1378/chest.93.3.580

13. Jones PW, Harding G, Berry P, Wiklund I, Chen WH, Kline Leidy N: Development and first validation of the COPD Assessment Test. Eur Respir J. 2009, 34:648-54. 10.1183/09031936.00102509

14. Miller MR, Hankinson J, Brusasco V, et al.: Standardisation of spirometry. Eur Respir J. 2005, 26:319-38. 10.1183/09031936.05.00034805

15. Charlson ME, Pompei P, Ales KL, MacKenzie CR: A new method of classifying prognostic comorbidity in longitudinal studies: development and validation. J Chronic Dis. 1987, 40:373-83. 10.1016/00219681(87)90171-8

16. Mannino DM, Tal-Singer R, Lomas DA, et al.: Plasma fibrinogen as a biomarker for mortality and hospitalized exacerbations in people with COPD. Chronic Obstr Pulm Dis. 2015, 2:23-34. 10.15326/jcopdf.2.1.2014.0138

17. Global Initiative for Chronic Obstructive Lung Disease . (2019). Accessed: June 14, 2021: https://goldcopd.org/.

18. Vilar R, Fish RJ, Casini A, Neerman-Arbez M: Fibrin(ogen) in human disease: both friend and foe . Haematologica. 2020, 105:284-96. 10.3324/haematol.2019.236901 
19. Lowe GD, Rumley A, Mackie IJ: Plasma fibrinogen. Ann Clin Biochem. 2004, 41:430-40. 10.1258/0004563042466884

20. Vestbo J, Agusti A, Wouters EF, et al.: Should we view chronic obstructive pulmonary disease differently after ECLIPSE? A clinical perspective from the study team. Am J Respir Crit Care Med. 2014, 189:1022-30. 10.1164/rccm.201311-2006PP

21. Han MK, Quibrera PM, Carretta EE, et al.: Frequency of exacerbations in patients with chronic obstructive pulmonary disease: an analysis of the SPIROMICS cohort. Lancet Respir Med. 2017, 5:619-26. 10.1016/S2213-2600(17)30207-2

22. Kim V, Criner GJ: The chronic bronchitis phenotype in chronic obstructive pulmonary disease: features and implications. Curr Opin Pulm Med. 2015, 21:133-41. 10.1097/MCP.0000000000000145

23. Miller BE, Tal-Singer R, Rennard SI, et al.: Plasma fibrinogen qualification as a drug development tool in chronic obstructive pulmonary disease. Perspective of the Chronic Obstructive Pulmonary Disease Biomarker Qualification Consortium. Am J Respir Crit Care Med. 2016, 193:607-13. 10.1164/rccm.201509$1722 \mathrm{PP}$

24. Cook DG, Cappuccio FP, Atkinson RW, et al.: Ethnic differences in fibrinogen levels: the role of environmental factors and the beta-fibrinogen gene. Am J Epidemiol. 2001, 153:799-806. 10.1093/aje/153.8.799

25. Undas A: How to assess fibrinogen levels and fibrin clot properties in clinical practice? . Semin Thromb Hemost. 2016, 42:381-8. 10.1055/s-0036-1579636

26. Dahl M, Tybjaerg-Hansen A, Vestbo J, Lange P, Nordestgaard BG: Elevated plasma fibrinogen associated with reduced pulmonary function and increased risk of chronic obstructive pulmonary disease. Am J Respir Crit Care Med. 2001, 164:1008-11. 10.1164/ajrccm.164.6.2010067

27. Engström G, Segelstorm N, Ekberg-Aronsson M, Nilsson PM, Lindgärde F, Löfdahl CG: Plasma markers of inflammation and incidence of hospitalisations for COPD: results from a population-based cohort study. Thorax. 2009, 64:211-5. 10.1136/thx.2008.102079

28. Kim S, Eliot M, Koestler DC, Wu WC, Kelsey KT: Association of neutrophil-to-lymphocyte ratio with mortality and cardiovascular disease in the Jackson Heart Study and Modification by the Duffy Antigen Variant. JAMA Cardiol. 2018, 3:455-62. 10.1001/jamacardio.2018.1042

29. Seemungal T, Harper-Owen R, Bhowmik A, et al.: Respiratory viruses, symptoms, and inflammatory markers in acute exacerbations and stable chronic obstructive pulmonary disease. Am J Respir Crit Care Med. 2001, 164:1618-23. 10.1164/ajrccm.164.9.2105011

30. Kunter E, Ilvan A, Ozmen N, et al.: Effect of corticosteroids on hemostasis and pulmonary arterial pressure during chronic obstructive pulmonary disease exacerbation. Respiration. 2008, 75:145-54. $10.1159 / 000097748$ 